\title{
Entornos virtuales online y diseño centrado en el usuario: un estudio de caso
}

口n

Juan López-Tarruella Maldonado, Carmen Llinares-Millán, Jaime Guixeres-Provinciale y Juan Luis Higuera-Trujillo

Universidad Politécnica de Valencia (España)

\section{DOI: http://dx.doi.org/10.6036/7905}

\section{INTRODUCCIÓN}

Un Entorno Virtual (EV) es un escenario tridimensional, interactivo y con visión en primera persona, generado por ordenador que simula un espacio existente 0 ficticio y en el que el usuario puede sentir presencia física. Esta definición comprende desde un simple videojuego 3D hasta lo que se conoce como Realidad Virtual. Los Entornos Virtuales, en sus distintas variantes (simuladores, serious games, Realidad Virtual...) se están usando en los últimos años en disciplinas tan diversas como la medicina, psicología, educación, cultura y ocio, ingeniería y arquitectura [1].

En el ámbito del diseño, los EVs se utilizan para la evaluación del uso y seguridad de las edificaciones durante el proceso constructivo y en el mantenimiento a lo largo de su vida útil. Así, se encuentran aplicaciones en la evaluación de accesibilidad de entornos urbanos, la simulación de evacuación de edificios en situaciones de emergencias, el entrenamiento de personal técnico en obra en cuestiones de seguridad y salud y manejo de instalaciones o la simulación de fases constructivas críticas en proyectos de gran complejidad [2].

Pero, quizá la mayor aceptación y desarrollo que está adquiriendo el uso de EVs es como herramienta de visualización y toma de decisiones durante las fases de diseño [3], ya que posibilita la observación en primera persona de espacios no construidos a un coste relativamente bajo, sin imposición de un punto de vista (como ocurre en fotografía), con capacidad de salvar limitaciones físicas (accesibilidad, iluminación ) y con la posibilidad de efectuar fácilmente modificaciones de diseño. En el ámbito del diseño centrado en el usuario se encuentran estudios basados en la presentación de propuestas (distribuciones, mobiliario ) al cliente para recoger sus opiniones y preferencias [4].
En este sentido, cabe destacar el campo de investigación denominado Evidence-Based Design, surgido hace pocas décadas y que, ubicado entre la ergonomía, la psicología ambiental y la ciencia experimental, trata de analizar cómo los espacios construidos afectan a las personas, con objeto de crear diseños orientados a las necesidades del usuario [5], especialmente en el ámbito hospitalario donde éste es más vulnerable. Habitualmente, estos estudios han presentado al sujeto estímulos visuales con las variables a analizar para, posteriormente, registrar su respuesta. En una situación ideal, los estímulos serían espacios reales donde el sujeto percibiera plenamente el espacio. En la práctica, estos estudios muestran al sujeto fotografías o infografías de los espacios [6], quedando la validez de las respuestas condicionadas a la capacidad de recreación espacial de unos usuarios que, muchas veces, son profanos en el ámbito de "imaginar espacios". Esta limitación resulta especialmente patente cuando se valoran sentimientos o emociones asociadas a la experiencia espacial ("cuánto me gusta", "cómo me hace sentir"...) que exigen del usuario una especial agudeza mental para evocar las sutilezas y sensaciones del espacio supuesto. En este sentido, en los últimos años ya se están utilizando Entornos Virtuales para presentar estos estímulos [3].

Un número cada vez mayor de estudios comparativos entre formatos de visualización corrobora que los EVs transmiten una mayor sensación de presencia física en el espacio simulado [7]. Sin embargo, estos trabajos no han analizado en profundidad el conjunto de emociones que el Entorno Virtual es capaz de generar. En este sentido cabría peguntarse ¿pueden los Entornos Virtuales utilizarse para estudiar las emociones que originaria la experiencia de un espacio real? Este es el objetivo del presente trabajo: comprobar si los Entornos Virtuales son una herramienta adecuada para analizar la respuesta emocional de las personas ante el espacio construido. Igualmente, resultaba de interés comprobar la idoneidad de una metodología de evaluación online, ya que permite el acceso a un mayor número de evaluadores.
Para ello se planteó un caso de estudio en el que se recogió mediante cuestionario online la valoración emocional de un conjunto de usuarios ante un espacio interior virtualizado en el que se modificó de forma aislada y controlada una variable independiente. El espacio seleccionado como entorno a evaluar fue una sala de lactancia, ya que armonizaba requisitos funcionales y emocionales (necesidad intimidad, seguridad, tranquilidad...). Además el uso real de este tipo de salas es eminentemente individual, lo cual eliminaba la necesidad de incorporar personajes virtuales que pudieran entorpecer una experiencia puramente espacial. Se escogió el color como elemento de diseño a estudiar, al ser un factor objetivo, cuantificable, modificable $y$, según la bibliografía, relacionado con la respuesta emocional [8], seleccionando ocho gamas cromáticas a partir de tonos equidistantes en la escala NCS.

Los resultados de este trabajo pueden resultar de interés para arquitectos por un doble motivo; en primer lugar, porque se comprueba la aptitud del uso de EVs Online en posteriores evaluaciones del factor emocional de futuros espacios; y en segundo lugar, porque se facilitan criterios de selección de gamas cromáticas adaptadas a las necesidades emocionales de las personas.

\section{MATERIAL Y MÉTODOS}

\subsection{CUESTIONARIO}

Se diseñó un cuestionario para ser respondido por el usuario durante la navegación por los distintos Entornos Virtuales. Tras responder afirmativamente a la pregunta filtro "¿Sabe usted que es y para qué sirve una sala de lactancia?" se solicitaban los datos demográficos del participante y se preguntaba mediante escalas tipo Likert de cinco puntos sobre seis cualidades del espacio, tres ejes emocionales y la valoración del espacio.

En primer lugar se preguntaba "Esta sala de lactancia me parece..." para los seis atributos del espacio Funcional / Acogedora / Con buen diseño / Amplia / SenciIla / Luminosa, obtenidos a partir de una muestra mayor de adjetivos sobre espacios sanitarios valorados por un conjunto de usuarios mediante Semántica Diferen- 
cial [9]. La ventaja de esta técnica es que permite extraer el esquema conceptual del observador.

En segundo lugar se preguntaba "Esta sala de lactancia me transmite..." para las tres emociones Placer - Bienestar/Relajación / Seguridad, adaptación a la evaluación del espacio del Modelo PAD (Pleasure/Arousal/Dominance) para descripción y medición de estados emocionales [10] propuesto por los psicólogos Mehrabian y Russell.

Por último se preguntaba "En términos generales, esta sala de lactancia a nivel ESTÉTICO me gusta..." y "Si tuviera que utilizar una sala de lactancia, me gustaría que fuera esta..." para obtener la valoración puramente estética y la global incluyendo funcionalidad.

\subsection{CREACIÓN DE LOS ENTORNOS} VIRTUALES

2.2.1. Modelado tridimensional del espacio

El espacio a evaluar constaba de una zona con tres puestos de lactancia con si-
Ilones y sus mesas auxiliares, un puesto independiente con mayor privacidad, una bancada con zona de preparación de biberones, lavamanos y cambia pañales, y acceso a una sala de uso restringido.

Se partió de un archivo 2D creado con AutoCAD 2010 conteniendo la planta que fue limpiado y preparado para su exportación al software de modelado tridimensional SketchUp 2015. Se decidió un nivel de detalle que permitiera un grado de realismo aceptable sin comprometer el tamaño de archivo. Una vez concluido el modelo tridimensional de la sala (Figura 1) se exportó en un archivo en formato FBX que es aceptado por el game engine Unity3Dv5, para la creación de los escenarios interactivos.

2.2.2. Aplicación de luces, colores y materiales

Con el modelo tridimensional importado en el game engine Unity3D, se creó una luz solar exterior y luminarias interiores. La iluminación indirecta, cuyo complejo cálculo no se puede realizar en tiempo real, se efectuó mediante lightmapping.

En cuanto al color, se decidió que cada una de las ocho gamas cromáticas seleccionadas a partir de tonos equidistantes en la escala NCS contuviera tres colores, manteniendo tono y saturación, y variando oscuridad al 5\%, 30\% y 60\%, utilizando el color claro en paredes, el medio en el suelo y un pilar, y el oscuro en puertas y muebles bajo encimera. El techo, los estores y el resto de elementos se mantuvieron en blanco o tonos neutros desaturados.

Para conseguir mayor efecto de tridimensionalidad y realismo se aplicaron ciertos efectos de materialidad; En las paredes se incluyó un normalmap de relieve simulando un acabado con cierta rugosidad. En el suelo se aplicó un mapa de textura granulada simulando pavimento vinílico. A los elementos panelados en madera lacada (puertas y mobiliario bajo bancada) se les aumentó el nivel de brillo especular. El resultado fueron ocho escenarios interactivos con iluminación, color y material aplicados. En la Figura 2 se muestran los ocho escenarios virtuales online utilizados
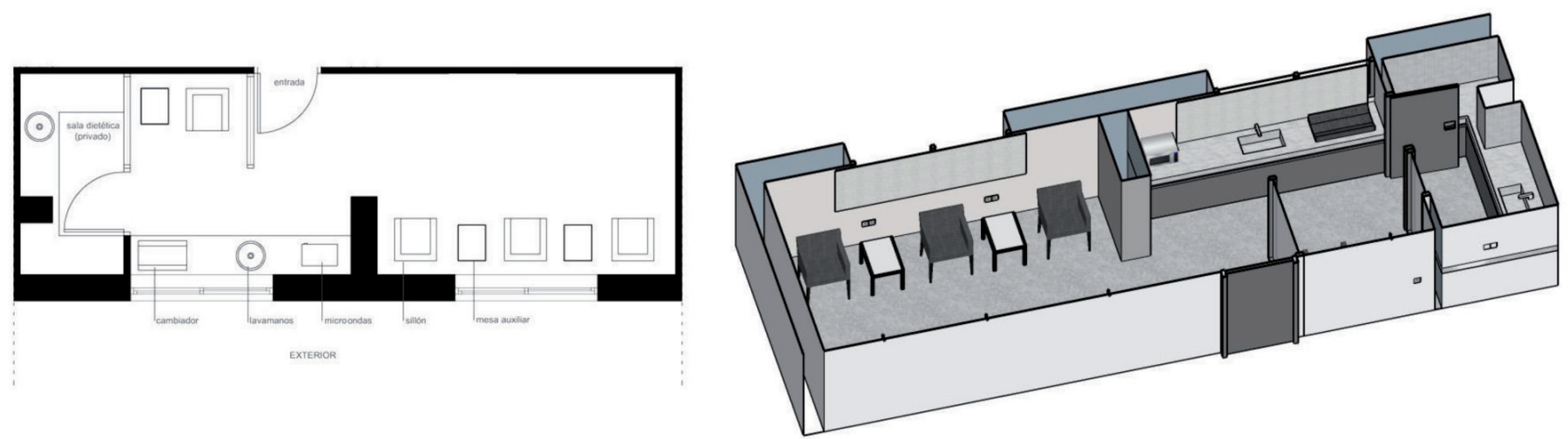

Fig. 1: Planta de la sala de lactancia hospitalaria y proceso de desarrollo del modelo tridimensional
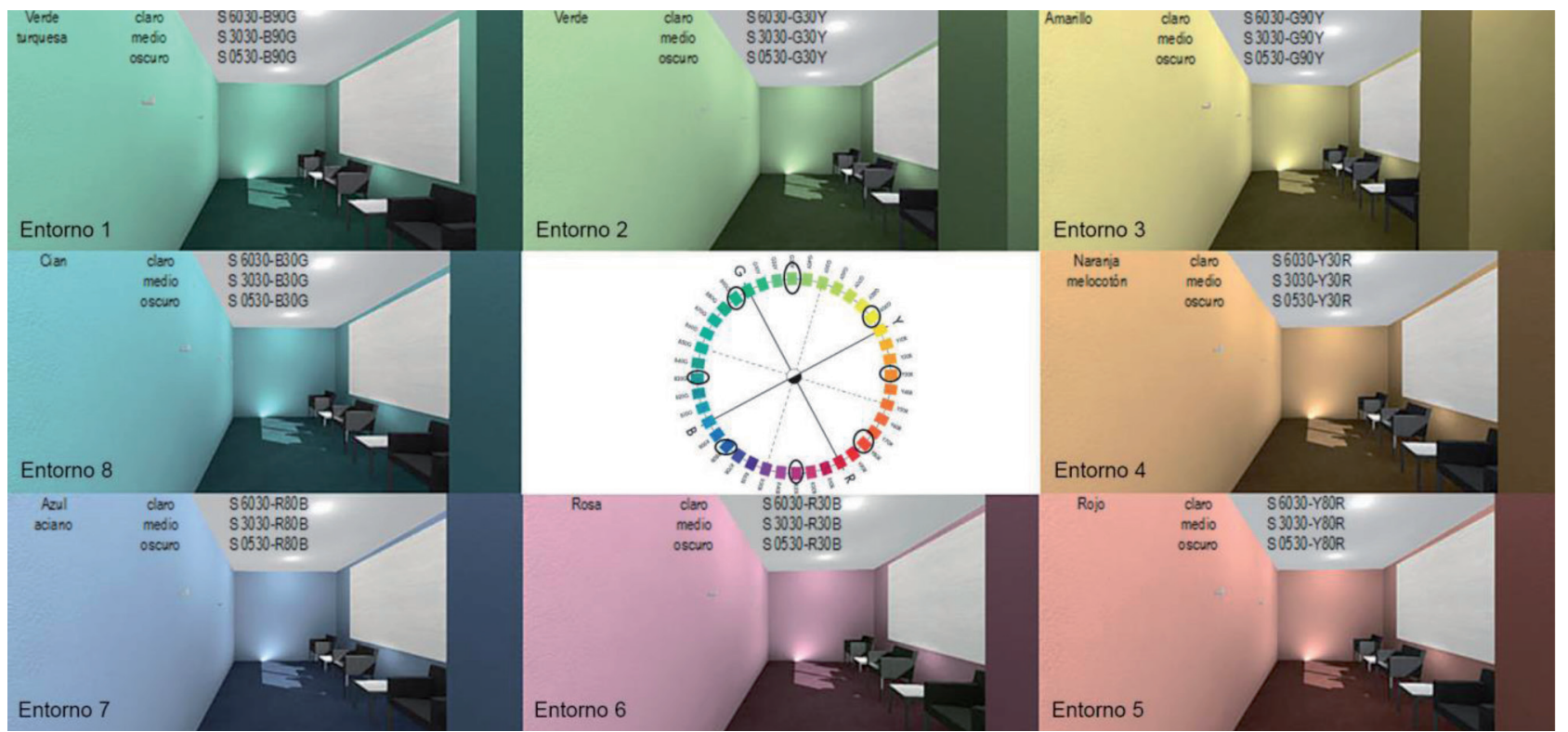

Fig. 2: Los ocho escenarios online en las distintas gamas cromáticas seleccionadas con sus códigos de color 
con la codificación de los colores utilizados según el estándar NCS.

2.2.3. Creación de los entornos navegables online

Una vez terminado el modelo virtual, se generaron los ocho entornos navegables (uno por color) en formato compatible con visualización en navegadores de internet mediante el plugin Unity Web Player. Los archivos fueron copiados en un servidor de internet y se vincularon al software de encuestas online de tal forma que a cada usuario se le presentara un entorno con color al azar.

\subsection{MUESTRA}

La muestra total estudiada fue de 72 participantes, 9 sujetos por cada uno de los ocho escenarios propuestos, a los que se les aleatorizaron los estímulos. El tamaño muestral se calculó considerando un nivel de confianza del 95\%, una varianza de 1 (según estudios similares de valoración del espacio con imágenes, y para una escala tipo Likert de 5 puntos) y un margen de error de 0,65. Las características de los sujetos se recogen en la Tabla I.

La selección de los encuestados se realizó, como se ha comentado, a través del propio cuestionario mediante la pregunta "¿Sabe usted que es y para qué sirve una sala de lactancia?" eliminando a aquellos sujetos que respondian de forma negativa. La difusión se realizó mediante la colaboración de diversas asociaciones relacionadas con la infancia y la lactancia. El vínculo difundido conducía a la encuesta online gestionada por la plataforma Survey Monkey.

\subsection{TRATAMIENTO ESTADÍSTICO} DE DATOS

Los datos obtenidos fueron tratados estadísticamente con el software SPSS v.22.0.

a. Análisis descriptivo de las valoraciones emocionales de los sujetos

En primer lugar se realizó un análisis descriptivo para detectar tendencias en los resultados. Los valores para cada variable se normalizaron a sus puntuaciones $z$ de forma que simplificara su interpretación comparativa, tanto entre colores como entre conceptos valorados.

\section{b. Análisis de diferencias significativas en función del perfil del sujeto}

Se comprobó mediante el Test de Shapiro-Wilk que las distribuciones no ajustaban a una distribución normal, por lo que se buscaron diferencias significativas en las respuestas entre perfiles, mediante los test no paramétricos Kruskal-Wallis (edades) y U de Mann-Whitney (género, hijos, y uso de sala de lactancia).

\section{c. Relación entre gamas cromáticas y la estructura conceptual}

A continuación se buscaron correlaciones estadisticamente significativas, mediante el coeficiente de correlación Rho de Spearman para muestras no paramétricas, entre colores (variable independiente), atributos del espacio, emociones percibidas y valoraciones.

\section{d. Obtención del espacio perceptual de los sujetos}

Con el objeto de agrupar conceptos eliminando la posible relación entre las variables estudiadas, se efectuó un análisis factorial que identificara factores subyacentes independientes. El método de extracción fue el análisis de componentes principales con rotación Varimax. El reducir las variables a dos factores integradores ortogonales permitió ordenar y representar gráficamente los valores medios de cada gama cromática en función de estos ejes.

\section{RESULTADOS}

a. Análisis descriptivo de las valoraciones emocionales de los sujetos

En los resultados se observa que los tonos 4 (naranja melocotón) y 7 (azul

\begin{tabular}{|c|c|c|c|c|c|c|c|c|c|c|c|}
\hline \multicolumn{3}{|c|}{ edad } & \multicolumn{3}{|c|}{ genero } & \multicolumn{3}{|c|}{ ¿Tiene hijos?: } & \multicolumn{3}{|c|}{$\begin{array}{l}\text { ¿Ha usado alguna vez } \\
\text { una sala de lactancia? }\end{array}$} \\
\hline & Frec. & $\%$ & & Frec. & $\%$ & & Frec. & $\%$ & & Frec. & $\%$ \\
\hline$<30$ años & 14 & $19 \%$ & hombre & 23 & $32 \%$ & no & 15 & $21 \%$ & no & 30 & $42 \%$ \\
\hline 30 a 40 años & 48 & $67 \%$ & mujer & 49 & $68 \%$ & si & 57 & $79 \%$ & si & 42 & $58 \%$ \\
\hline 40 a 50 años & 10 & $14 \%$ & & & & & & & & & \\
\hline Total & 72 & $100 \%$ & Total & 72 & $100 \%$ & Total & 72 & $100 \%$ & Total & 72 & $100 \%$ \\
\hline
\end{tabular}

Tabla l: Características de los sujetos encuestados

\begin{tabular}{|c|c|c|c|c|c|c|c|c|c|c|c|}
\hline \multirow[b]{2}{*}{ escenario } & \multicolumn{6}{|c|}{ Atributos del espacio } & \multicolumn{3}{|c|}{ Emociones } & \multicolumn{2}{|c|}{ Valoración } \\
\hline & Funcional & Acogedora & $\begin{array}{c}\text { Buen } \\
\text { diseño }\end{array}$ & Amplia & Sencilla & Luminosa & Relajación & Bienestar & Seguridad & $\begin{array}{c}\text { valor } \\
\text { estético }\end{array}$ & $\begin{array}{l}\text { valor } \\
\text { global }\end{array}$ \\
\hline 1 &,- 258 &,- 162 &, 033 &, 143 &,- 290 &,- 464 &,- 379 &,- 473 &, 131 &,- 463 &,- 291 \\
\hline 2 &,- 178 &, 061 &,- 177 &,- 027 &, 114 & ,222 &,- 379 &,- 020 &,- 008 & ,104 & ,170 \\
\hline 3 &, 573 &,- 162 & ,292 & ,316 & ,290 & ,348 &,- 379 &,- 290 &,- 083 &,- 005 &, 108 \\
\hline 4 &, 227 & ,340 & ,292 &,- 086 & ,255 & ,235 & ,745 &, 537 &,- 056 & ,302 & ,220 \\
\hline 5 &,- 437 &,- 306 &,- 829 &,- 163 &,- 088 &,- 139 & ,351 &,- 310 &,- 025 &,- 463 &,- 769 \\
\hline 6 &,- 056 &,- 288 &,- 161 &,- 220 &,- 769 &,- 504 &,- 251 &,- 005 &,- 083 &,- 005 &,- 032 \\
\hline 7 & , 101 & ,215 & ,292 & ,048 & 290 & 206 & ,387 & ,138 &, 053 & 275 & ,108 \\
\hline 8 &,- 150 & ,240 & ,171 &, 021 &, 114 &,- 106 &,- 379 & ,309 & ,162 & ,079 & ,443 \\
\hline
\end{tabular}


aciano) obtienen, en general, las mejores valoraciones, no obteniendo ningún valor debajo de la media en ninguno de los apartados evaluados, y los tonos 3 (amariIlo) y 8 (cian) presentan tendencia positiva aunque no tan marcada. Por el contrario, los tonos 5 (rojo), 6 (rosa) y 1 (verde turquesa) acumulan las peores valoraciones. En la tabla de contingencia (Tabla II) se han marcado los valores negativos en rojo y los extremos en negrita para facilitar su examen.

Análisis de diferencias significativas en función del perfil del sujeto

Destacan diferencias estadísticamente significativas en el nivel 0,05 entre grupos de usuarios con hijos y sin hijos en los aspectos Acogedora (sig. 0,024), Luminosa (sig. 0,002), Relajación (sig. 0,001), valor estético (sig. 0,044) y valor global (sig. $0,002)$, siendo en todos los casos inferior la valoración de usuarios con hijos. No se encontraron diferencias en los demás aspectos.

\section{b. Relación entre gamas cromáticas y la estructura conceptual}

En cuanto a la relación entre atributos en sí, y respecto a las valoraciones, se produjeron múltiples correlaciones estadisticamente significativas, entre las que destacan las cuatro con mayores índices de correlación; bienestar / valor estético $\left(r_{s}=, 829, \rho=, 2 \times 10^{-19}\right)$, acogedora / valor estético $\left(r_{s}=, 803, \rho=, 2 \times 10^{-17}\right)$, bienestar / acogedora $\left(r_{s}=, 762, \rho=, 7 \times 10^{-15}\right)$ y valor estético / valor global $\left(r_{s}=, 700, \rho=, 7 \times 10^{-12}\right)$.

Por otro lado, en cuanto a la relación entre gamas cromáticas con atributos y valoraciones, se encontraron correlaciones positivas entre color 3 (amarillo) con Funcional $\left(r_{s}=, 234, \rho=, 048\right)$, color 4 (melocotón) con Relajación $\left(r_{s}=, 320, \rho=, 006\right)$, y correlaciones negativas entre color 5 (rojo) con Buen diseño $\left(r_{s}=-, 309, \rho=, 008\right)$, Valor estético $\left(r_{s}=-, 245, \rho=, 038\right)$ y Valor global $\left(r_{s}=-, 332, \rho=, 004\right)$ y color 6 (rosa) con Sencilla $\left(r_{s}=-, 288, \rho=, 014\right)$.

\section{c. Obtención del espacio perceptual de los sujetos}

Los resultados del análisis factorial (Tabla III) indican que los nueve conceptos evaluados podrian ser reducidos a dos factores capaces de explicar el $65 \%$ de la varianza total obtenida en las respuestas del cuestionario;

- Factor 1: Englobando los conceptos

\begin{tabular}{c|cc} 
& Factor 1 funcionalidad & Factor 2 bienestar \\
\hline \% varianza explicada & 0,51 & 0,14 \\
Alpha de Crombach & 0,878 & 0,897 \\
\hline Amplia &, 828 &,- 023 \\
Funcional &, 761 &, 277 \\
Buen diseño &, 733 &, 293 \\
Sencilla &, 658 &, 167 \\
Luminosa &, 521 &, 457 \\
Relajación &,- 141 &, 863 \\
Bienestar &, 401 &, 757 \\
Acogedora &, 554 &, 690 \\
Seguridad &, 521 &, 648
\end{tabular}

Tabla III: Análisis factorial
Amplia, Funcional, Sencilla, Buen diseño y Luminosa. Correspondería con el componente relativo a la funcionalidad y explicaría un 51\% de la varianza total. La consistencia del espacio perceptual fue verificada mediante Alfa de Cronbach, resultando una fiabilidad considerable de la escala $(0,878)$.

- Factor 2: Englobando los conceptos Relajación, Placer/Bienestar, Acogedora y Seguridad. Correspondería con el componente relativo al bienestar y explicaría un 14\% de la varianza total. Alfa de Cronbach 0,897.

La reducción de las nueve variables evaluadas a dos ejes independientes y ortogonales facilita la representación gráfica de la respuesta del usuario ante la variación del color en el espacio, organizando los valores medios de cada gama cromática en función de las nuevas variables; factor 1 (funcionalidad) y factor 2 (bienestar) (figura 3).

La distribución de los colores en la gráfica permite apreciar la cercanía existente entre tonos verdosos (colores $1 \mathrm{y}$ 2), rojizos (5 y 6) y azulados (7 y 8) e, igualmente, la agrupación que se produce en los tonos fríos (colores 1, 2, 7 y 8), lo cual testifica la integridad de los resultados. Se observa como los tonos rojizos tienden a valoraciones negativas en el eje funcionalidad y neutras en bienestar. Los tonos fríos, por el contrario, se mantienen neutros en el eje funcionalidad y se extienden a lo largo del eje bienestar desde el verde hasta el azul aciano. En cuanto a los ocres, el tono melocotón puntúa neutro en funcionalidad y muy positivamente en bienestar mientras que el amarillo lo hace muy positivamente en funcionalidad y negativamente en bienestar.

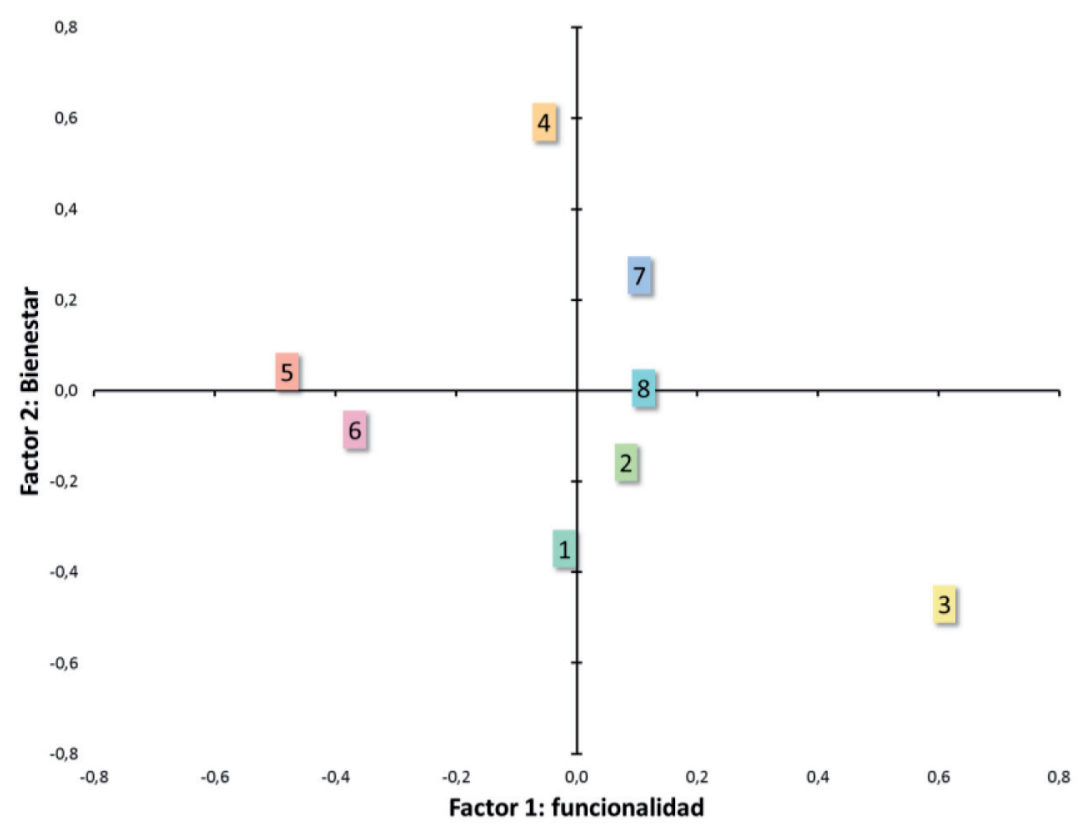

Fig. 3: Representación de las gamas cromáticas en función de los ejes "funcionalidad" y "bienestar" 


\section{DISCUSIÓN Y CONCLUSIONES}

El presente trabajo ha puesto de manifiesto la aptitud del uso de Entornos Virtuales y cuestionarios online como herramienta para la evaluación de la componente emocional del espacio, tanto en su proceso de creación y difusión, como en la respuesta obtenida por parte de los usuarios.

Las contribuciones más relevantes a nivel de resultados son las siguientes:

Por una parte, se confirma que las valoraciones tienden a polarizarse, inclinándose los tonos ocres ( 3 y 4 ) y azulados (7 y 8) a obtener las mejores valoraciones en los distintos aspectos evaluados frente a los rojizos (5 y 6), que agrupan claramente los peores resultados, como se aprecia en la Tabla II. Sorprendentemente, la gama cromática verde ( 1 y 2 ) tiende a valoraciones negativas pese a ser el color predominante en la naturaleza, cuya visión se relaciona habitualmente con la inducción al bienestar y relajación. Esto podría radicar en que este tono, en espacios sanitarios, evoca al tradicional verde quirúrgico por lo que, en vez de favorecer la calma, refuerza la sensación de estrés propia del procedimiento hospitalario.

Asimismo, se han puesto de manifiesto relaciones fuertemente significativas entre el atributo del espacio "acogedor", la emoción "bienestar" y la valoración estética positiva del espacio. Retornando a la Tabla II, se comprueba que el color 4 (melocotón) obtiene sistemáticamente la mejor puntuación en todos estos apartados, además de una correlación estadísticamente significativa con la emoción "relajación". Por ello, parece oportuno interpretar que, para transmitir sensación de bienestar, los espacios sanitarios han de percibirse como acogedores, para lo cual deberian diseñarse principalmente en tonos ocres o azules, evitando en lo posible los rojizos y el "verde hospital".

Por otra parte, resulta coherente, pero significativo, que las nueve variables evaluadas hayan sido reducidas a una escala bidimensional definida por lo emocional y lo funcional del espacio, donde se aprecia que los colores similares obtienen valores próximos (figura 3). Esto nos lleva a considerar de interés la dupla "funcionalidad bienestar" para futuras evaluaciones de la percepción del espacio por parte del usuario. La dispersión producida en los tonos ocres (colores 3 y 4) podría indicar una mayor variabilidad de respuesta del usuario en este rango cromático, motivando a futuros trabajos en este espectro.

Por otro lado, las diferencias encontradas entre el grupo de sujetos con y sin hijos demuestra la necesidad de segmentar estos dos grupos de sujetos, aspecto a tener en cuenta en futuros estudios.

En cuanto a las limitaciones del presente trabajo hay que considerar que los resultados obtenidos son específicos del uso y configuración del espacio concreto estudiado. Igualmente, la muestra utilizada (72 usuarios) es relativamente baja, siendo necesario ampliarla para obtener mayor consistencia en los resultados. Por otra parte, hay que mencionar que ciertas variables del diseño, como el confort térmico o acústico, no pueden realizarse siguiendo la metodología propuesta mediante Entornos Virtuales online, requiriendo experimentos presenciales.

Los resultados aquí presentados, tanto en la evaluación de gamas cromáticas como en el uso de Entornos Virtuales, pueden resultar de utilidad para aquellos arquitectos que deseen tener en cuenta las preferencias del usuario durante las fases de diseño. La evolución de la tecnología de uso doméstico está abriendo nuevas posibilidades a la hora de presentar y recoger información del ciudadano. En un mundo cada vez más conectado e interactivo parece indispensable aprovechar esta oportunidad en provecho de un diseño centrado en las personas.

Futuras líneas de investigación pretenden, (1), comparar la respuesta del usuario ante distintos formatos de presentación de escenarios (imagen, imagen panorámica $360^{\circ} \times 180^{\circ}$, Entorno Virtual...) y soportes (pantalla 2D, cascos de Realidad Virtual...), (2) comparar los resultados obtenidos mediante cuestionario, con la respuesta objetiva o involuntaria del sujeto a través de sistemas de medición psicofisiológico (EEG, HRV, GSR) y, (3) ampliar el catálogo de variables de diseño: materiales de revestimiento, presencia de naturaleza...

\section{PARA SABER MÁS}

[1] Burdea G C and Coiffet $P$, Virtual reality technology, vol. 12, no. 6. John Wiley \& Sons, Inc., 2003.

[2] Li M, "Applications of virtual reality technology in construction industry," 2010 2nd International Conference on Computer Engineering and Technology, vol. 4, p. V4-223-V4-227, 2010, DOI: http://dx.doi. org/10.1109/ICCET.2010.5486354.

[3] Bullinger H-J J, Bauer W, Wenzel G, et al., "Towards user centred design (UCD) in architecture based on immersive virtual environments," Computers in Industry, vol. 61, no. 4, p. 372-379, May 2010, DOI: http:// dx.doi.org/10.1016/j.compind.2009.12.003.

[4] Rebelo F, Noriega P, Duarte E, et al., "Using Virtual Reality to Assess User Experience.," Human Factors, vol. 54, no.
6, p. 964-982, 2012, DOI: http://dx.doi. org/10.1177/0018720812465006.

[5] Huisman E R C M, Morales E, van Hoof J, et al., "Healing environment: A review of the impact of physical environmental factors on users," Building and Environment, vol. 58, p. 70-80, 2012, DOI: http://dx.doi. org/10.1016/j.buildenv.2012.06.016.

[6] Dijkstra K, Pieterse M E, and Pruyn a. TH, "Individual differences in reactions towards color in simulated healthcare environments: The role of stimulus screening ability," Journal of Environmental Psychology, vol. 28 no. 3, p. 268-277, 2008, DOI: http://dx.doi. org/10.1016/j.jenvp.2008.02.007.

[7] Westerdahl B, Suneson K, Wernemyr C, et al., "Users' evaluation of a virtual reality architectural model compared with the experience of the completed building," Automation in Construction, vol. 15, no. 2, p. 150-165, 2006, DOI: http://dx.doi. org/10.1016/j.autcon.2005.02.010.

[8] Yildirim K, Akalin-Baskaya a., and Hidayetoglu M L, "Effects of indoor color on mood and cognitive performance," Building and Environment, vol. 42, no. 9, p. 3233-3240, 2007, DOI: http://dx.doi. org/10.1016/j.buildenv.2006.07.037.

[9] Osgood C E, "THE NATURE AND MEASUREMENT OF MEANING," Psychological Bulletin, vol. 49, no. 3, p. 227, 1952, DOI: http://dx.doi.org/10.1037/h0021468.

[10] Mehrabian, A., \&t Russell J a., An approach to environmental psychology. 1974.

\section{AGRADECIMIENTOS}

Esta investigación ha sido apoyada por el Ministerio de Economía y Competitividad. España (proyecto TIN2013-45736-R). 